\title{
Preparation of diethylaminoethyl hollow fibres for high-flow liquid chromatography
}

\author{
Alexander C.R. Tsuei and Victor C. Yang \\ College of Pharmacy, University of Michigan, Ann Arbor. MI 48109-1065, USA \\ (Received 12 March 1990; accepted 9 April 1990)
}

\begin{abstract}
A novel anion-exchange column for fast-flow liquid chromatography was developed. The column was prepared by immobilizing diethylaminoethyl groups on to the inner walls of regenerated cellulose hollow fibres. A detailed investigation was conducted of the parameters affecting diethylaminoethyl immobilization such as the $\mathrm{NaOH}$ concentration, the reaction temperature, the diethylaminoethyl concentration and the reaction span. Based upon the findings, a protocol to optimize the preparation of the diethylaminoethyl hollow fibres was established. The diethylaminoethyl hollow fibres prepared adsorbed $186 \pm 30 \mathrm{mg}$ of albumin $/ \mathrm{g}$ fibres.
\end{abstract}

Kerwords: Cellulose, fibres, liquid chromatography

lon-exchange chromatography based on porous resins has been used extensively in the separation and purification of proteins and other biomaterials from complex mixtures of proteins $^{1}$. Unfortunately, due to the restricted physical and mechanical properties of these resins, separation using the resin-bedded column is often hindered by the limited flow rate and operating pressure. This paper presents a new anion-exchange column which can be operated at almost unrestricted flow rates and operating pressures. The column was prepared by immobilizing diethylaminoethyl (DEAE) groups on to regenerated cellulose hollow fibres. The effects of variables such as the concentration of the activating agent (i.e. $\mathrm{NaOH}$ ), the reaction temperature, the concentration of DEAE in the coupling solution and the reaction span were examined. From the results, a protocol to optimize the preparation of DEAE hollow fibres (DEAE-HFs) was developed. The protocol indicated the use of $0.6 \mathrm{~N}$ of $\mathrm{NaOH}$ for the activation of the hollow fibres, an $\mathrm{NaOH}$ concentration between 0.25 and $0.6 \mathrm{~N}$ in the coupling solution, a temperature of $63^{\circ} \mathrm{C}$ during the pretreatment and the coupling process, $3 \mathrm{~g}$ of the amine salt in the coupling solution and four coupling runs during the coupling process. The DEAE-HFs prepared were capable of adsorbing $186 \pm 30 \mathrm{mg}$ of albumin $/ \mathrm{g}$ fibre.

The new, hollow fibre-based ion-exchange column offers several advantages over the standard particulate columns. First, it obviates the tedious and time-consuming

Correspondence to Dr. V.C. Yang steps for the preparation, loading and packing of the gelbased columns. Second, the hollow fibre column provides an almost unrestricted flow rate for sample adsorption and desorption, as opposed to the limited operating pressures required by conventional resins. Third, the hollow fibre column can be easily scaled-up from a laboratory scale to a preparative/production scale without pressure drop and bed compression concerns which hinder scale-up when using particulate chromatography matrices. Fourth, the hollow fibre matrix permits the direct contact of the mobile phase components with the active surface area, alleviating diffusion dependence and eliminating phase equilibration encountered when using the standard chromatography matrices.

\section{MATERIALS AND METHODS}

\section{Materials}

2-Chlorotriethylamine hydrochloride was purchased from Fluka Chemical Corporation (Ronkonkoma, NY, USA). BioRad dye reagent was purchased from Bio-Rad Laboratories (Richmond, CA, USA). Cellulose hollow fibres were obtained from a clinically used Model 1500 hemodialyzer manufactured by Travenol Laboratories (Deerfield, IL, USA). Water was distilled, then deionized with a mili- $Q^{\text {TM }}$ water system albumin solution was prepared by dissolving $2.5 \mathrm{mg}$ of bovine serum albumin in $1 \mathrm{ml}$ of $10 \mathrm{mM} \mathrm{Na}_{2} \mathrm{HPO}_{4}$ buffer at $\mathrm{pH} 7.6$.

(c) 1990 Butterworth-Heinemann Ltd. 0142-9612/90/090734-04 


\section{Methods}

Assays. Albumin concentration was measured according to the method of Bradford ${ }^{2}$. The anion-exchange capacity of the DEAE-HFS was estimated based on the amount of albumin adsorbed per gram of the fibres.

Fibrication of the hollow bundle. Cellulose hollow fibres were collected in a bundle $(0.8-1 \mathrm{~g}$ in weight and $16.25 \mathrm{~cm}$ in length). At each end of the bundle, a piece of 2 in $(5 \mathrm{~cm})$ Tygon tubing inside diameter $1 / 8$ in $(31 \mathrm{~mm})$ was mounted to serve as the connector. The junction was glued with rubber cement (Sanford Corp., Bellwood, IL, USA) and sealed with epoxy adhesives (Devcon Corp, Danvers, MA, USA). The bundle was then ready to use.

DEAE immobilization. DEAE residues were immobilized on to the inner walls of the hollow fibres, according to a modified procedure of Peterson and Sober ${ }^{3}$. The immobilization process consisted of two steps: (1) pretreatment of the cellulose hollow fibres with sodium hydroxide and (2) coupling of the DEAE groups on to the activated hollow fibres.

Pretreatment of the hollow fibres. To activate the hollow fibre bundle, $\mathrm{NaOH}$ solutions at concentrations ranging from 0.6 to $2.0 \mathrm{~N}$ were used. The solution $(50 \mathrm{ml})$ was placed into a reservoir, heated with a heating plate to a desired temperature, then circulated through the bundle for $200 \mathrm{~s}$ at a flow rate of $60 \mathrm{ml} / \mathrm{min}$. To maintain a constant reaction temperature, the bundle was also heated with an infrared heatlamp ( $250 \mathrm{~W}$ and $115 \mathrm{~V}$ ) during the pretreatment and the coupling process.

Coupling of DEAE on to the activated fibres. The coupling solution was prepared by adding concentrated $\mathrm{NaOH}$ to the aqueous solution of 2-chlorotriethylamine hydrochloride. Unless otherwise stated, a weight of $3 \mathrm{~g}$ of 2-chlorotriethylamine hydrochloride was used in each batch of the coupling solution. To examine the effect of $\mathrm{NaOH}$ on DEAE immobilization, various amounts of $\mathrm{NaOH}$ were used to prepare the coupling solution. The final $\mathrm{NaOH}$ concentration in the coupling solution was calculated from the amount of $\mathrm{NaOH}$ added, assuming that each mole of the 2-chlorotriethylamine hydrochloride salt would require an equal mole of $\mathrm{NaOH}$ to convert it to the neutral 2-chlorotriethylamine. For each coupling run, the pretreated bundle was circulated with $50 \mathrm{ml}$ of coupling solution for $200 \mathrm{~s}$ at a flow rate of $60 \mathrm{ml} / \mathrm{min}$. Unless otherwise stated, the coupling process was repeated for four times with four coupling runs and each coupling run was done with a freshly prepared coupling solution. The bundle was suction-dried using an aspirator equipped with a water suction apparatus. The bundle was then washed subsequently with $300 \mathrm{ml}$ of $1 \mathrm{~N} \mathrm{NaCl}, 80 \mathrm{ml}$ of $1 \mathrm{~N} \mathrm{HCl}, 100 \mathrm{ml}$ of $1 \mathrm{~N} \mathrm{NaOH}, 100 \mathrm{ml}$ of distilled water and $100 \mathrm{ml}$ of $10 \mathrm{~mm}$ phosphate buffer at $\mathrm{pH} 7.6$. Before the albumin adsorption experiments, the DEAE-HF bundle was further rinsed with $200 \mathrm{ml}$ of the same $10 \mathrm{~mm}$ sodium phosphate buffer.

Zeta potential measurement. The DEAE-bound hollow fibres were air dried, frozen in liquid nitrogen and ground into a fine powder with a pestle. The powder was then suspended in water and the suspension sonicated with a Branson E-Module sonicator for $1 \mathrm{~h}$ to obtain a colloidal

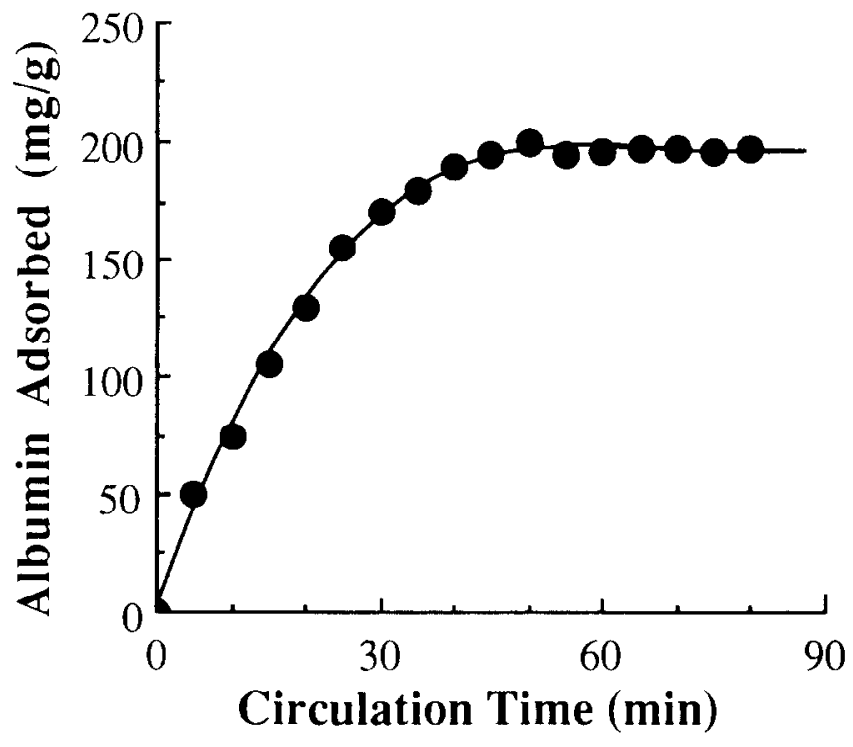

Figure 1 A representitive albumin adsorption-time plot for the DEAE-HF bundle.

suspension. The zeta potential of the colloidal particles was measured using laser Zee Model 501 zetameter.

Albumin adsorption. The anion exchange capacity of the DEAE-HF was represented by the amount of albumin adsorbed per gram of the fibre. Figure 1 shows a representitive albumin adsorption-time plot. The albumin solution, $120 \mathrm{ml}$ $(2.5 \mathrm{mg} / \mathrm{ml})$, was placed in a reservoir and circulated through the hollow fibre bundle $(0.8-1 \mathrm{~g})$ at a flow rate of $60 \mathrm{ml} / \mathrm{min}$. At various time intervals, a sample was withdrawn from the reservoir and measured for the residual albumin concentration. The circulation was continued until the final albumin concentration reached a plateau. The anionexchange capacity $\left(A_{\text {cap }}\right)$ of the fibre was then calculated according to the equation: $A_{\text {cap }}=\left(C_{f}-C_{i}\right) \times V / W_{t}$, where $C_{f}$ and $C_{i}$ represented the initial and final albumin concentration, respectively, $V$ was the volume of the solution and $W_{t}$ was the net weight of the hollow fibres. Each adsorption experiment was conducted in duplicate, and the mean value was used to represent the $A_{\text {cap }}$ value.

\section{RESULTS AND DISCUSSION}

Figure 2 shows the mechanism of DEAE immobilization. The hydrochloride salt of diethylaminoethyl chloride was converted to the active form of $N, N$-diethylaziridinium chloride by the addition of $\mathrm{NaOH}$, then covalently linked to the hydroxyl groups on the glucose residues of the cellulose polymer. It has been reported that the chemical availability of the $-\mathrm{OH}$ groups on the C-2, C-3 and C- 6 positions of the glucose repeating unit is strongly dependent on the involvement of these groups in the formation of inter- or intramolecular hydrogen bonds ${ }^{4}$. The major purpose of using $\mathrm{NaOH}$ during the coupling procedure is thus to disrupt the formation of hydrogen bonds through a swelling process, so that the chemical availability of the hydroxyl groups can be enhanced.

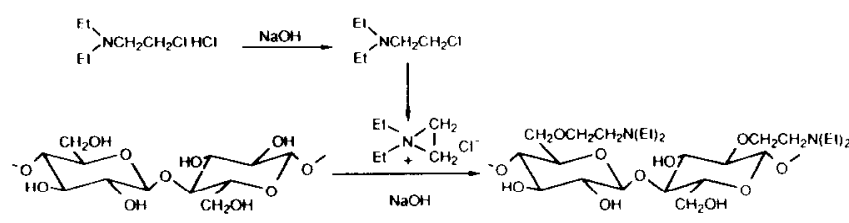

Figure 2 Mechanism of DEAE immobilization on cellulose hollow fibres. 
The effect of the $\mathrm{NaOH}$ concentration employed in the pretreatment step was studied. A $\mathrm{NaOH}$ concentration above $1.5 \mathrm{~N}$ imposed a significant deterioration of the fibre structures, as indicated by the occlusion of flow inside the fibre lumens. Occlusion might result from two mechanisms: (1) the inner diameter $(150-200 \mu)$ of the hollow fibre was reduced, due to the penetration of the alkali solution into the amorphous regions of cellulose, and (2) the mobility of water on the fibre surface was reduced, due to the formation of hydrogen bonds between the $-\mathrm{OH}$ groups on the fibre and the eluent water cluster ${ }^{5}$. Reducing the $\mathrm{NaOH}$ concentration to under $1 \mathrm{~N}$ appeared to retain the flow characteristics of the fibres. Scanning electron microscopy (SEM) data revealed, however, that only at a $\mathrm{NaOH}$ concentration of $0.6 \mathrm{~N}$ or below that the fibres would absolutely maintain their initial structures. $\mathrm{A} \mathrm{NaOH}$ concentration of $0.6 \mathrm{~N}$ was therefore selected to activate the fibre bundle, because this $\mathrm{NaOH}$ concentration would provide the best activation without compromising the physical integrity of the fibres.

The effect of the $\mathrm{NaOH}$ concentration in the coupling solution on DEAE immobilization was also studied and the results are summarized in Table 1.The degree of DEAE immobilization was indirectly reflected by the amount of albumin adsorbed by each gram of the fibre. Sodium hydroxide concentrations under testing were held to under $1 \mathrm{~N}$ to assure the retention of the flow characteristics of the fibre. As shown in Table 1, the amount of albumin adsorbed per gram of fibre remained almost unchanged (i.e. about $200 \mathrm{mg}$ albumin/g fibre) as the $\mathrm{NaOl} / \mathrm{l}$ concentration in the coupling solution was varied from 0.85 to $0.25 \mathrm{~N}$. However, a stiff drop in albumin adsorption occurred when the $\mathrm{NaOH}$ concentration was reduced to $<0.25 \mathrm{~N}$. A slight change of the $\mathrm{NaOH}$ concentration from 0.25 to $0.15 \mathrm{~N}$ resulted in a drop of albumin adsorption of $>70 \%$. The results were consistent with those obtained from the zeta potential measurements. Bundles prepared at $\mathrm{NaOH}$ concentrations between 0.25 and $0.85 \mathrm{~N}$ gave nearly identical values in zeta potential, suggesting that the bundles carried similar amount of surface charges i.e. a similar amount of the positively charged DEAE groups. Reducing the $\mathrm{NaOH}$ concentration from 0.25 to $0.05 \mathrm{~N}$ resulted in a marked drop in the zeta potential value. Albumin adsorption by the fibres was apparently not due to the $\mathrm{NaOH}$ treatment, but rather to the presence of the DEAE groups on the fibres,

Tahle 1 Effect of sodium hydroxide concentration on DEAE immobilization Bundles were prepared using $0.6 \mathrm{~N}$ of $\mathrm{NaOH}$ for pretreatment, four coupling runs, $3 \mathrm{~g}$ of 2-chlorotriethylamine hydrochloride salt in each batch of coupling solution and a reaction temperature of $62^{\circ} \mathrm{C}$

\begin{tabular}{lll}
\hline $\begin{array}{l}\mathrm{NaOH} \text { concentration } \\
(\mathrm{N})\end{array}$ & $\begin{array}{l}\text { Albumin adsorbed }\left(A_{\text {cap }}{ }^{\mathrm{a}}\right) \\
(\mathrm{mg} / \mathrm{g} \text { fibre })\end{array}$ & Zeta potential ${ }^{\mathrm{b}}$ \\
\hline 0.85 & 230 & +63.5 \\
0.75 & 167 & +62.5 \\
0.65 & 151 & +65.8 \\
0.55 & 214 & +65.0 \\
0.45 & 154 & +62.5 \\
0.35 & 187 & +63.0 \\
0.25 & 200 & +65.8 \\
0.15 & 64 & +59.4 \\
0.05 & 10 & +53.6 \\
\hline
\end{tabular}

${ }^{a}$ The $A_{\text {cao }}$ value (i.e. the anion exchange capacity) of the fibre was calculated according to the equation: $A_{\text {cap }}=C_{f}-C_{i} \times V / W_{t}$. Each adsorption experiment was conducted in duplicate and data were represented as the mean value.

${ }^{b}$ Zeta potential was measured in duplicate. Data were presented as the mean value.
Table 2 Effect of reaction temperature on DEAE immobilization. Bundles were prepared using $0.6 \mathrm{~N}$ of $\mathrm{NaOH}$ during the pretreatment step, $3 \mathrm{~g}$ of 2 chlorotriethylamine hydrochloride salt in each batch of coupling solution and $0.25 \mathrm{~N}$ of final $\mathrm{NaOH}$ concentration in the coupling solution

\begin{tabular}{ll}
\hline $\begin{array}{l}\text { Reaction temperature } \\
\left({ }^{\circ} \mathrm{C}\right)\end{array}$ & $\begin{array}{l}\text { Albumin adsorbed }\left(A_{\text {cap }}{ }^{a}\right) \\
(\mathrm{mg} / \mathrm{g} \text { fibre })\end{array}$ \\
\hline 73 & 10 \\
62 & 210 \\
52 & 54 \\
44 & 10 \\
23 & 0
\end{tabular}

${ }^{a}$ The $A_{\text {cap }}$ value (i.e. the anion exchange capacity) of the fibre was calculated according to the equation: $A_{\text {cap }}=C_{f}-C_{i} \times V / W_{\mathrm{t}}$. Each adsorption experiment was conducted in duplicate and data were represented as the mean value.

since bundles treated with $\mathrm{NaOH}$ solution containing no DEAE did not elicit any measurable albumin adsorption (data not shown). The $\mathrm{NaOH}$ concentration of $0.25 \mathrm{~N}$ was deemed to be the minimal concentration required in the coupling solution to achieve optimal DEAE immobilization.

It should be pointed out that because of the rather limited changes in zeta potential, this value was not proportionally related to the amount of albumin which was adsorbed by the DEAE-HFs, so that albumin adsorption by DEAE-HFs could not be predicted. The zeta potential value could, however, be used to index the success of DEAE immobilization. A zeta potential value below 59 would normally provide the DEAE-HFs with a low albumin adsorption capacity of $<50 \mathrm{mg} / \mathrm{g}$ fibre.

Table 2 shows the effect of temperature on DEAE immobilization. The temperature used in the pretreatment and the coupling processes was controlled to be the same and ranged from 23 to $73^{\circ} \mathrm{C}$. As shown in Table 2, increasing the temperature from 23 to $63^{\circ} \mathrm{C}$ resulted in an increase in the amount of DEAE immobilized, indicated by the related increase in the amount of albumin adsorbed. This temperature-dependent increase in DEAE immobilization could be due to the increase in the immobilization kinetics at higher temperatures. Further elevation of the reaction temperature to $73^{\circ} \mathrm{C}$, however, resulted in a dramatic drop in albumin adsorption from 210 to $10 \mathrm{mg} / \mathrm{g}$ fibre. Gentile and co-workers reported that the accessibility of the hydroxyl groups on the cellulose polymer dropped at high temperature due to the recrystallization of the amorphous regions of cellulose. The drop in DEAE immmobilization at high reaction temperatures appeared to be consistent with their findings. The temperature of $62^{\circ} \mathrm{C}$ therefore seemed to be the best reaction temperature for DEAE immobilization.

The effect of DEAE concentration in the coupling solution, and the number of coupling runs used during the coupling process are given in Table 3, which shows that the bundle which was prepared using $3 \mathrm{~g}$ of the amine salt in each of the coupling batch and four or five coupling runs offered the highest albumin adsorption, 210 and $236 \mathrm{mg}$ of albumin/g fibre, respectively. Using five coupling runs, however, resulted in a partial occlusion of the bundle after it was repeatedly circulated with the albumin solution. Tab/e 3 reveals that the bundle treated with five coupling runs possessed an exceptionally high value in zeta potential, +76 . This high value in zeta potential implied that the bundle was overcoated with DEAE molecules. As a consequence, there was an overloading of albumin on the bundle, which eventually blocked the flow of the bundle.

Based upon the above results, an optimal procedure 
Table 3 Effect of DEAE concentration and the number of coupling runs on DEAE immobilization. Bundles were prepared using $0.6 \mathrm{~N}$ of $\mathrm{NaOH}$ during the pretreatment step, $0.25 \mathrm{~N}$ of final $\mathrm{NaOH}$ concentration in the coupling solution, and a reaction temperature of $62^{\circ} \mathrm{C}$

\begin{tabular}{llll}
\hline $\begin{array}{l}\text { Amount of } \\
\text { DEAE }(g)\end{array}$ & $\begin{array}{l}\text { Number of } \\
\text { runs }\end{array}$ & $\begin{array}{l}\text { Albumin } \\
\text { adsorbed } \\
\left.\left(A_{\text {caj }}\right)^{\prime}\right)(\mathrm{mg} / \mathrm{g} \text { fibre })\end{array}$ & $\begin{array}{l}\text { Zeta } \\
\text { potential }{ }^{\mathrm{b}}\end{array}$ \\
\hline 3 & 1 & 0 & +49 \\
3 & 2 & 0 & +51 \\
3 & 3 & 50 & +59 \\
3 & 4 & 210 & +66 \\
3 & 5 & $236^{\mathrm{c}}$ & +76 \\
6 & 1 & 24 & -50 \\
6 & 2 & 30 & - \\
6 & 3 & 60 & $>75$ \\
6 & 4 & $-{ }^{\mathrm{d}}$ & \\
\hline
\end{tabular}

${ }^{a}$ The $A_{\text {cap }}$ value (i.e. the anion exchange capacity) of the fibre was calculated according to the equation: $A_{\text {cap }}=C_{\mathrm{f}}-C_{\mathrm{i}} \times V / W_{\mathrm{t}}$. Each adsorption experiment was conducted in duplicate and data were represented as the mean value.

'Zeta potential was measured in duplicate. Data were presented as the mean value.

'The bundle was partially occluded following repeated albumin adsorption.

${ }^{d}$ The bundle was fully occluded following repeated albumin adsorption.

for achieving the highest degree of DEAE immobilization without compromising the physical integrity of the hollow fibres was obtained. The procedure suggested the use of $0.6 \mathrm{~N}$ of $\mathrm{NaOH}$ for the activation of the hollow fibres, a NaOH concentration between 0.25 and $0.6 \mathrm{~N}$ in the coupling solution, a temperature of $65^{\circ} \mathrm{C}, 3 \mathrm{~g}$ of the DEAE amine salt in the coupling solution, and four coupling runs during the coupling process. The DEAE-HF prepared adsorbed about $200 \mathrm{mg}$ of albumin $/ \mathrm{g}$ fibre.

Unlike the standard particulate column which contains gel pores and the stationary phase, the hollow fibre-based ion-exchange column does not contain the stationary phase and thus alleviates diffusion dependence. Mobile phase components are in direct contact with the surface area as the solution passes through the fibre lumen. Alternating the elution flow rate would not, therefore, affect the resolving capabilities of the column. However, to be practical, the column should be more suitable for adoption of the stepwise elution procedures than the linear elution gradients.

The new ion-exchange hollow fibres ofter several advantages over the particulate ion-exchange matrices. One advantage is that the run preparation time was dramatically reduced using the hollow fibre column since the column loading/packing is completely eliminated and the column equilibration/ regeneration is significantly reduced. Another advantage is that hollow fibres are the constructing materials for an artificial kidney and thus almost non-resistant to flow. The pressure drop in a hollow fibre bed is extremely low and the column can therefore be operated at very high linear velocities. The third advantage is the presence of linear scaleup capabilities for the hollow fibre-based column. The column can be prepared to any size and length without the concerns of the flow rate versus pressure drop trade-offs that hinder scale-up when using standard chromatography matrices.

The only shortcoming of the hollow fibre-based ionexchange column is the lack of large surface area per volume for solute adsorption. This can, however, be overcome by two different approaches. One is to adopt dead-end geometry ${ }^{6,7}$. In this case, the feed solution is forced across the hollow fibre wall and the adsorption will occur in a very thinpacked bed of very large cross-sectional area. The other approach is to attach spacer arms on to the inner walls of the fibre, followed by the attachment of DEAE molecules to these arms. As a result, the inner lumen of the fibre will consist of multiple, fur-like layers of DEAE for solute adsorption. Experiments related to the latter approach are now being conducted in our laboratory.

\section{ACKNOWLEDGEMENTS}

This study was supported by the National Institutes of Health grant R29-HL38353 and a Whitaker Foundation Biomedical Engineering Grant.

\section{REFERENCES}

1 Ion Exchange Chromatography: Principles and Methods, Pharmacia, Uppsala, Sweden, 1982

2 Bradford, M., A rapid and sensitive method for the quantitation of microgram quantities of protein utilizing the principle of protein-dye binding, Anal. Biochem. 1976, 72, 248-254

3 Peterson, E.A. and Sober, H.A., Chromatography of proteins. I. Cellulose ion-exchange adsorbents, J. Am. Chem. Soc. 1956, 78, 751-755

4 Bertoniere, N.R. and Zeronian, S.H., Chemical characterization of cellulose, in The Structures of Cellulose, (Ed. R.H. Atalla), American Chemical Society, Washington D.C., USA, 1987, pp 255-271

5 Gentile, V.M., Schroeder, L.R. and Atalla, R.H., Physical structure and alkaline degradation of hydrocellulose, in The Structures of Cellulose, (Ed. R.H. Atalla), American Chemical Society, Washington D.C., USA, $1987,272-291$

6 Haggin, J., Gas membrane technology applied to liquid separation of biologicals, C\&EN News, March 13, 1989, 20-21

7 Ding, H., Yang, M.C., Schisla, D. and Cussler, E.L., Hollow-fibre liquid chromatography, AlChE J. 1989, 35, 814-820 